\title{
Growth, water relations and physiological processes of starfruit (Averrhoa carambola L) plants under root growth restriction
}

\begin{abstract}
Starfruit (Averrhoa carambola L., Clone B17) plants were grown in two levels of root zone volume $(3.36 \mathrm{~m} 3$ or $0.68 \mathrm{~m} 3)$ and two levels of water availability (well watered or $30 \%$ of field capacity). Root restriction inhibited the growth of starfruit plants as indicated by the reduction in leaf and root growth, and to a greater extent under water stress conditions. Under well-watered conditions, root growth restriction resulted in a slight reduction in leaf water potential. Regardless of watering regimes, root growth restriction causes an increase in leaf diffusive resistance, which consequently contributes to the reduction in leaf photosynthetic rate. The beneficial effect of root growth restriction in plants grown under well watered conditions is to hasten flower initiation.
\end{abstract}

Keyword: Root restriction; Averrhoa carambola; Growth; Flowering; Leaf water potential; Stomatal resistance; Photosynthetic rate 